\author{
Research Article
}

\title{
A COMPARATIVE CLINICAL STUDY ON 'JARA' WITH RESPECT TO AGEING PROCESS WITH ASHWAGANDHA RASAYANA AND MATRA BASTI
}

\author{
Singh Binod Kumar ${ }^{1 *}$, Bharkher D.L2 \\ *1Asst. Professor \& Head, Dept. of Panchakarma, Ayurveda Campus, IOM, Tribhuvan University, Kathmndu, Nepal. \\ 2Professor \& Head, Dept. of Kayachikitsa, Executive Director, T.U. Ayurveda Teaching Hospital, IOM, Kathmandu, Nepal.
}

\begin{abstract}
The problem of ageing is experienced by all the countries. According to World Health statistics the life expectancy of Nepalese people has risen from 58.91 years to 67.86 years (1996 to 2015). Ageing is emerging issue in Nepal as well as global. Its tempo is expected to be unexpectedly fast as mortality continues to decline and life expectancy continues to increase. Ayurveda the science of life has observed ageing as a Jara avastha, which is a later phase of life, it is natural, inevitable phenomenon, in which maximum decline of bodily elements that may become as major cause of disability and functional dependency requiring services that affect many sectors of economy, health, security, income, housing, transportation etc. Jara chikitsa has been mentioned as one independent Anga in Ashtanga Ayurveda where Rasayana therapy is capable to impede the ageing process and to delay the degenerative process in the body. In this study we measured the effectiveness of Ashwagandha rasayana and Matra basti and compared with the Ashwagandha Rasayan only in Jara avastha. A total of thirty elderly patients were selected and divided in two groups A and B, given them either Ashwagandha Rasayana with Matra basti or Ashwagandha rasayana in prescribed doses for 45 days. Changes in the subjective complaints, objective parameters of the patients and appearance of adverse events were also evaluated. Both the groups provided better results on the chief complaints But, comparison in between both the groups is insignificant, that may be due to small sample size.
\end{abstract}

KEYWORDS: Ageing, Ashwagandha, Jara, Matra basti.

\section{INTRODUCTION}

Though growing old is a part of life cycle, a strong group of above 60 years of age is building up to cause further challenge to the medical world. In Nepal the older population accounts for about 8.1 percent of the total population and a majority (over 6 out of 10 ) of them are in their sixties ${ }^{[1]}$, elderly people are defined as those above 60 years of age as per the WHO definition and they are addressed as Senior Citizens.[2] The Central Bureau of Statistics (CBS) of Nepal reported that the percentage of population aged 60 years and above increased from $5.0 \%$ during 1952- 54, $5.8 \%$ in 1991, 6.5\% during 2001 and $8.1 \%$ in 2011 accounting for an increase of nearly 2.46 million. ${ }^{[3]}$ Recently, worldwide population ageing have been considered one of the most important demographic phenomena. It is the product of clear decreases in birth and mortality rates and an increase in life expectancy, which is reflected in the socio-economical progress of countries. ${ }^{[4]}$ The worldwide population aged 60 years and older will surpass from approximately 770 million in 2010 to an estimated one billion in 2020 , and $20.0 \%$ of these people will be concentrated in developing countries, Thus, it needs to be addressed and proper attention should be given to the elderly people.[5]

Geriatrics is a vast branch of medicine dealing exclusively with the problems of ageing and the diseases of elderly like, degenerative deficits, dementia, Parkinson's disease, C.O.P.D, Insomnia etc. The World Health Organization (WHO) very appropriately declared the calendar year 1999 as the year of Elderly People - Senior Citizens, to focus on the growing problems of the ageing people. This offers an opportunity to gather the scattered matter spread over many Ayurvedic classics and other sources of information.

The problem is how to keep old people physically fit and how to make them useful to society. This calls for intensive research into the causes which leads to ageing.

Ayurveda the science of life has observed ageing as a graceful phenomenon. Old age and its health problems were of great concern even for Acharyas during Vedic period. That was the reason why Jara Chikitsa has been mentioned as one independent Anga in Ashtanga Ayurveda. It implies delaying the process of ageing, prevention and managing the problems of old age by using of Rasayana drugs. This Jara or Rasayana chikitsa has widely emphasized promoting, protecting and extending youthful state and thus curtailing ageing process. It is therapy which gives longevity, enhances memory and intellect, maintains good health and restores youth, complexion, body colour, voice. It also gives strength to the physical and to the sense and cognitive organs. ${ }^{6]}$ So, there is responsibility to come forward to take preventive measures and treatment aspects for the old age problem.

All the Rasayana drugs have properties like Medhya, Balya, Dhatupushtikara, Brumhaniya, Jivaniya, and Agnivardhaka etc. and also have proven pharmacological 
Singh Binod Kumar et al. 'Jara' with Respect to Ageing Process with Ashwagandha Rasayana and Matra Basti

actions, such as: Adaptogens, Antioxidant, Immunostimulants, Antistress, Nootropics, Anabolics, Tonics and other geriatric properties. Thus, Rasayana formulations described in the classics have very good composition with maximum properties on demand. Ashwagandha rasayana and Matra basti is selected for the study because Ashwagandha (Withania somnifera) is explained as Tikta, Katu- kashaya rasayukta, Ushna veerya, Madhur vipaka it is Vata kapha shamaka, Sukrala, Balya, Rasayana like Jara vikara nashaka, Dhatu vardhaka etc.[7] Rasayana promotes digestion and metabolism, thereby proper production to Rasa dhatu and elimination of toxic metabolites is achieved, which in turn promote Dhatu poshana. Prabhava of Rasayana does the equilibrium of Tridosha, which is responsible to restore normal physical and mental activities in the same way Matrabasti is a very unique therapeutic procedure because of its preventive, health promotive, rejuvenative properties. It is applicable in all Vatavyadhis (neurological deficits). Matra basti is most indicated procedure in Vrudhas due to property of Balya, Vatahara and Agni Sandhukshana ${ }^{[8]}$ By using Aswagandha rasayana and Matra basti the quality of life of elderly may be improved and ageing process may be delayed and can be postponed. So, the topic "A Comparative Clinical Study on 'Jara' With Respect to Ageing Process with Ashwagandha Rasayana and Matra Basti "was been undertaken for study.

\section{OBJECTIVE OF THE STUDY}

\section{General Objectives}

- To find out a safe and effective intervention in the management of Jaraavastha, that could improve the quality of life in the elderly.

\section{Specific}

- To evaluate the effectiveness of Ashwagandha rasayana and Matra basti in improving quality of life in elderly persons.

- To compare the effectiveness of both Ashwagandha rasayana and Matra basti in improving quality of life in elderly persons.

\section{MATERIALS AND METHODS}

\section{Materials}

A. Drugs

1. Group A - Ashwagandha churna

2. Group B - Ashwagandha churna Mahanarayan tail, Shatapushpa churna and Saindhava lavana.

Source of drugs - Ashwagandha churna and Mahanarayan tail are prepared as per classical references

B. Thirty old age persons

C. Instruments

1. Syphagnomanometer

2. Weighing machine

3. Clinical thermometer

4. Stop watch

5. Glycerine syringe of $60 \mathrm{ML}$

6. Catheter no 9.

\section{Sources}

The literary sources of the study were collected from the classical texts of Ayurveda, published journals, modern texts, international editions and internet. The elderly persons were taken from OPD and IPD of TU Ayurveda Teaching Hospital, Kirtipur and Medical camps conducted by the institute.

\section{Sampling method}

Minimum of 30 elderly persons were selected excluding drop outs. They were assigned into two groups consisting of 15 persons in each group viz., Group A and Group B. Statistical analysis were done on subjective parameters such as Agnimandhya, Malabadhata, Bala Kshaya, Grahana Kshaya, etc. and objective parameters such as Haemoglobin, Blood Pressure Etc. as mentioned in Ayurvedic and modern texts by 'Student's t-test'.

\section{Inclusive Criteria}

1) Elderly persons with classical symptoms of Jara such as Agnimandya, Bala Kshaya etc.

2) Both sexes

3) Age group in between 60 to $70 y r$.

\section{Exclusive Criteria}

1) Elderly persons suffering from any other systemic disorders.

2) Age above 70yrs.

3) Senile psychosis.

4) Elderly persons who are non co-operative.

\section{Study Design}

\section{Group A}

Drug: Ashwagandha churna

Dose: 5 gm B.D.

Method: Oral administration

Anupana: Sarkara added Sukhoshna dugdha

Duration: 45 days

Follow up: $15^{\text {th }}$ Days

\section{Group B:}

Drug: Ashwagandha churna 5 gm BD with Sarkara added Sukhoshna Dugdha as Anupana and $60 \mathrm{ML}$ of Mahanarayana tail Matra Basti.

Investigations were done for both the groups before and after the treatment.

\section{Investigations}

- Hemoglobin.

- Blood pressure

- Weight

\section{ASSESMENT CRITERIA AND THEIR GRADINGS}

\section{Subjective variables}

1) Agni mandya (Loss of Appetite)

Grade 1: Normal Appetite.

Grade 2: Diminished Appetite.

Grade 3: Loss of interest in taking food.

Grade 4: Aversion towards food.

\section{2) Mala baddhata (Constipation)}

Grade 1: No Constipation.

Grade 2: Regular bowel movements, consistency hard, excessive straining, and prolonged defecation time.

Grade 3: 3-5 Bowel movements per week, consistency hard. 
Grade 4: Less than 3 Bowel movements per week, consistency hard.

3) Balakshaya (Strength)

Grade 1: Normal strength.

Grade 2: Movement against some resistance only.

Grade 3: Movement against gravity only.

Grade 4: Movement with gravity eliminated only.

Grade 5: Palpable contraction or flicker.

Grade 6: No movement.

\section{4) Grahana kshaya (Loss of Grasping power)}

Person was asked to withhold the cuff of sphygmomanometer in folding manner for maximum time at maximum mercury level.

Grade 1: Can withhold more than $2 \mathrm{~min}$.

Grade 2: Can withhold in between $61 \mathrm{sec}$. to $2 \mathrm{~min}$.

Grade 3: Can withhold in between 30-60 sec.

Grade 4: Can withhold less than $30 \mathrm{sec}$.

\section{5) Nidra nasha (Insomnia)}

Grade 1: Adequate night sleep 6-8 hours.

Grade 2: Inadequate night sleep 4-5 hours

Grade 3: Inadequate night sleep 2-3 hours.

Grade 4: Inadequate night sleep 1-2 hours or no sleep.

\section{6) Shwasa (Exertional Dyspnoea)}

Grade 1: Dyspnoea on unaccustomed exertion.

Grade 2: Dyspnoea on accustomed Exertional work.

Grade 3: Dyspnoea on routine activities like moving about in the house, going to toilet, having bath.

Grade 4: Dyspnoea on rest.

7) Sarva kriya cha asamartha (Functional impairment)

Grade 1: Can perform routine work. No pain in joints of extremities.

Grade 2: Mild pain and stiffness in joints, walks with limping but without support.

Grade 3: Painful joints, walk without support, difficulty in performing daily routine.

Grade 4: Painful joints, Totally unable to perform daily routine.

\section{OBJECTIVE VARIABLES}

\section{Systolic Blood Pressure}

Grade 1 100-130 $\mathrm{mm}$ of $\mathrm{Hg}$.

Grade 2 132-140 $\mathrm{mm}$ of $\mathrm{Hg}$

Grade $3142-150 \mathrm{~mm}$ of $\mathrm{Hg}$

Grade 4 152-160 mm of $\mathrm{Hg}$

Grade 5 above 160

\section{Diastolic Blood Pressure}

Grade $170-90 \mathrm{~mm}$ of $\mathrm{Hg}$.

Grade 2 92-100 mm of $\mathrm{Hg}$.

Grade 3 102-110 mm of Hg.

Grade 4 above $110 \mathrm{~mm}$ of $\mathrm{Hg}$.

\section{Weight}

Grade 1 - 70kg and above
Grade 2 - 65.1-70kg.

Grade 3 - $160.1-65 \mathrm{~kg}$.

Grade 4 - 55.1-60 kg

Grade 5 - 50.1-55 kg

Grade 6 - 45.1-50 kg.

Grade 7 - 40.1-45 kg.

Grade 8 - below 40 kg.

\section{Haemoglobin}

Grade 1 - 14gm\% above

Grade 2 - 12.1- 14 gm \%

Grade 3 - 10.1 -12 gm\%

Grade 4- 8.1-10 gm.\%

Grade 5 - less than $8 \mathrm{gm} \mathrm{\%}$

\section{Assessment on clinical improvement}

Clinical improvement of the disease was based on improvement in the clinical finding and reduction on the severity of the symptoms of the disease grading for the clinical improvement for individual variables.

Grading for the clinical improvement for individual variables.

1. CD: Clinically deteriorated i.e. increase in severity score against the initial score.

2. CS: Clinically stable, i.e. severity score remains as against the initial score.

3. CI-1: encouraging i.e. I degree reduction in the severity score, against Initial score, i.e. reduction from mild-normal, Moderate-Mild and Severe- moderate.

4. CI-2: Good i.e. 2 degree reduction in the severity score, against the score, i.e. reduction from moderate-normal, severe-mild.

5. CI-3: excellent i.e. 3 degree reduction in the severity score, against the Initial Score, i.e. severe- normal.

Grading for clinical improvement of overall severity:

1. CD: Clinically deteriorated i.e. increase in severity score against the initial Score.

2. CS: Clinically stable, i.e. severity score remains as against the initial score.

3. CI-1: Clinically encouraging ( $1-6$ degree reduction in severity score).

4. CI-2: Clinically good i.e. (7 - 12 degree reduction in severity score).

5. CI-3: Clinically excellent i.e. (More than 13 degree reduction in severity score).

\section{Statistical analysis}

The data was collected from both groups before, after treatment and at the end of the follow up and statically analyzed by using ' $t$ ' test with the consultation of biostastician.

\section{OBSERVATION AND RESULTS}

Total 30 persons were selected for the clinical study and they were randomly allocated to Group A, Group $B$ respectively. The observations for the present study were done as. 
Singh Binod Kumar et al. 'Jara' with Respect to Ageing Process with Ashwagandha Rasayana and Matra Basti Generalized Observations

Table 1: Distribution of Patients Based on Agnimandya Before Treatment

\begin{tabular}{|l|l|l|l|l|}
\hline \multirow{2}{*}{ Agnimandya } & \multicolumn{2}{|l|}{ No. of Pt. from Group } & \multirow{2}{*}{ Total $(\mathbf{n}=30)$} & \multirow{2}{*}{ Percentage } \\
\cline { 2 - 5 } & A & B & & \\
\hline Grad -4 & 0 & 0 & 0 & $0 \%$ \\
\hline Grade-3 & 3 & 3 & 6 & $20 \%$ \\
\hline Grade-2 & 12 & 12 & 24 & $80 \%$ \\
\hline Grade-1 & 0 & 0 & 0 & $0 \%$ \\
\hline
\end{tabular}

Table 2: Distribution of Patients Based on Malabaddhata Before Treatment

\begin{tabular}{|l|l|l|l|l|}
\hline \multirow{2}{*}{ Malabaddhata } & \multicolumn{2}{|l|}{ No. of Pt. from Group } & \multirow{2}{*}{ Potal (n=30) } & \\
\cline { 2 - 3 } & A & B & & $0 \%$ \\
\hline Grad -4 & 0 & 0 & 0 & $30 \%$ \\
\hline Grade-3 & 4 & 5 & 9 & $53.3 \%$ \\
\hline Grade-2 & 7 & 9 & 16 & $16.6 \%$ \\
\hline Grade-1 & 4 & 1 & 5 & \\
\hline
\end{tabular}

Table 3: Distribution of Patients Based on Balakshaya Before Treatment

\begin{tabular}{|l|l|l|l|l|}
\hline \multirow{2}{*}{ Balakshaya } & \multicolumn{2}{|l|}{ No. of Pt. from Group } & \multirow{2}{*}{ Total $(\mathbf{n}=\mathbf{3 0})$} & \multirow{2}{*}{ Percentage } \\
\cline { 2 - 5 } & A & B & & \\
\hline Grad -4 & 0 & 0 & 0 & $0 \%$ \\
\hline Grade-3 & 1 & 0 & 1 & $3.3 \%$ \\
\hline Grade-2 & 13 & 15 & 28 & $93.3 \%$ \\
\hline Grade-1 & 1 & 0 & 1 & $3.3 \%$ \\
\hline
\end{tabular}

Table 4: Distribution of Patients Based on Grahanakshaya Before Treatment

\begin{tabular}{|l|l|l|l|l|}
\hline \multirow{2}{*}{ Grahanakshaya } & \multicolumn{2}{|l|}{ No. of Pt. from Group } & \multirow{2}{*}{ Total (n=30) } & \multirow{2}{*}{ Percentage } \\
\cline { 2 - 5 } & $\mathrm{A}$ & $\mathrm{B}$ & 0 & 0 \\
\hline Grad -4 & 0 & 0 & 4 & $13.3 \%$ \\
\hline Grade-3 & 2 & 2 & 22 & $73.3 \%$ \\
\hline Grade-2 & 11 & 11 & 4 & $13.3 \%$ \\
\hline Grade-1 & 2 & 2 & 0 & \\
\hline
\end{tabular}

Table 5: Distribution of Patients Based on Nidranasha Before Treatment

\begin{tabular}{|l|l|l|l|l|}
\hline \multirow{2}{*}{ Nidranasha } & \multicolumn{2}{|l|}{ No. of Pt. from Group } & \multirow{2}{*}{ Total $(\mathbf{n}=\mathbf{3 0})$} & \multirow{2}{*}{ Percentage } \\
\cline { 2 - 3 } & A & B & 0 & 0 \\
\hline Grad -4 & 0 & 0 & 0 & $10 \%$ \\
\hline Grade-3 & 1 & 2 & 25 & $83.3 \%$ \\
\hline Grade-2 & 14 & 11 & 2 & $6.6 \%$ \\
\hline Grade-1 & 0 & 2 & 25 & 0 \\
\hline
\end{tabular}

Table 6: Distribution of Patients Based on Shwasa Before Treatment

\begin{tabular}{|l|l|l|l|l|}
\hline \multirow{2}{*}{ Grahanakshaya } & \multicolumn{2}{|l|}{ NO of Pt. from Group } & \multirow{2}{*}{ Total (n=30) } & \multirow{2}{*}{ Percentage } \\
\cline { 2 - 5 } & A & B & & 0 \\
\hline Grad -4 & 0 & 0 & 0 & $13.3 \%$ \\
\hline Grade-3 & 3 & 1 & 4 & $83.3 \%$ \\
\hline Grade-2 & 11 & 14 & 25 & $3.3 \%$ \\
\hline Grade-1 & 1 & 0 & 1 & . \\
\hline
\end{tabular}

Table 7: Distribution of Patients Based on Sarvakriya Su Cha Asamartha Before Treatment

\begin{tabular}{|c|c|c|c|c|}
\hline \multirow{2}{*}{$\begin{array}{l}\text { Sarvakriya Su Cha } \\
\text { Asamartha }\end{array}$} & \multicolumn{2}{|c|}{ NO of Pt. from Group } & \multirow[t]{2}{*}{ Total $(n=30)$} & \multirow[t]{2}{*}{ Percentage } \\
\hline & $\mathrm{A}$ & $\mathrm{B}$ & & \\
\hline Grad -4 & 0 & 0 & 0 & 0 \\
\hline Grade-3 & 3 & 11 & 14 & $46.6 \%$ \\
\hline Grade-2 & 9 & 3 & 12 & $40 \%$ \\
\hline Grade-1 & 3 & 1 & 4 & $13.3 \%$ \\
\hline
\end{tabular}

Table 8: Systolic Blood Pressure Wise Distributions

\begin{tabular}{|l|l|l|l|l|l|}
\hline S.No. & Systolic B.P & Group-A & Group-B & Total (n=30) & Percentage \\
\hline 1 & $100-120 \mathrm{~mm}$ of Hg & 7 & 8 & 15 & $50 \%$ \\
\hline 2 & $122-140 \mathrm{~mm}$ of Hg & 5 & 4 & 9 & $30 \%$ \\
\hline 3 & $142-160 \mathrm{~mm}$ of Hg & 3 & 3 & 6 & $20 \%$ \\
\hline
\end{tabular}


Int. J. Ayur. Pharma Research, 2016;4(8):1-11

Table 9: Diastolic Blood Pressure Wise Distributions

\begin{tabular}{|l|l|l|l|l|l|}
\hline S.No. & \multicolumn{1}{|c|}{ Diastolic B.P } & \multicolumn{1}{|c|}{ Group-A } & \multicolumn{1}{|c|}{ Group-B } & \multicolumn{1}{|c|}{ Total $(\mathbf{n}=\mathbf{3 0})$} & \multicolumn{1}{|c|}{ Percentage } \\
\hline 1 & $70-90 \mathrm{~mm}$ of Hg & 11 & 12 & 23 & $76.6 \%$ \\
\hline 2 & $92-100 \mathrm{~mm}$ of Hg & 4 & 3 & 7 & $23.3 \%$ \\
\hline 3 & $102-110 \mathrm{~mm}$ of Hg & 0 & 0 & 0 & $0 \%$ \\
\hline
\end{tabular}

Table 10: Weight Wise Distributions

\begin{tabular}{|l|l|l|l|l|l|}
\hline S.No. & \multicolumn{1}{|c|}{ Weight in K.G } & Group-A & Group-B & \multicolumn{1}{|c|}{ Total $(\mathbf{n}=\mathbf{3 0})$} & \multicolumn{1}{c|}{ Percentage } \\
\hline 1 & Less than $40 \mathrm{Kg}$. & 0 & 1 & 1 & $3.3 \%$ \\
\hline 2 & $41-60 \mathrm{Kg}$. & 6 & 7 & 13 & $43.3 \%$ \\
\hline 3 & $61-80 \mathrm{Kg}$. & 9 & 6 & 15 & $50 \%$ \\
\hline 4 & More than $80 \mathrm{Kg}$, & 0 & 1 & 1 & $3.3 \%$ \\
\hline
\end{tabular}

Table 11: Hemoglobin wise Distributions

\begin{tabular}{|l|l|l|l|l|l|}
\hline S.No. & \multicolumn{1}{|c|}{ Hemoglobin } & Group-A & Group- B & Total (n=30) & \multicolumn{1}{c|}{ Percentage } \\
\hline 1 & $08-10 \mathrm{gm} \%$ & 1 & 0 & 1 & $3.3 \%$ \\
\hline 2 & $10.1-12 \mathrm{gm} \%$ & 4 & 3 & 7 & $23.3 \%$ \\
\hline 3 & $12.1-14 \mathrm{gm} \%$ & 6 & 10 & 16 & $53.3 \%$ \\
\hline 4 & $14.1-16 \mathrm{gm} \%$ & 4 & 2 & 6 & $20 \%$ \\
\hline
\end{tabular}

\section{Observations for Individual Group}

Group - A

$>$ All the patients were presenting with varied degree of Lakshanas.

$>$ The patients were given Ashwagandha churna 5 gm BD along with Sarkara added Sukhoshna dugdha as Anupana for 45 days.

$>$ Patients were comfortable during and end of treatment.

$>$ No adverse effects were seen.

$>$ All the patients came regularly for the post treatment follow up.

Group- B

$>$ All the patients were presenting with varied degree of Lakshanas.

$>\quad$ The patients were given Ashwagandha churna 5 gm BD with Sarkara added Sukhoshna dugdha as Anupana for 45 days and Mahanarayan tail Matra basti for 8 days.

$>$ Patients were comfortable during and end of treatment.

$>$ No adverse effects were seen.

$>$ All the patients came regularly for the post treatment follow up.

The Response of the Treatment for Individual Group (\%) After Treatment

Table 12: Subjective Parameters

\begin{tabular}{|c|c|c|c|c|c|c|}
\hline Variables & Group & \begin{tabular}{|l|} 
CI-3 \\
\end{tabular} & CI-2 & CI-1 & CS & \begin{tabular}{|l|} 
CD \\
\end{tabular} \\
\hline \multirow[t]{2}{*}{ Agnimandhya } & $\mathrm{A}$ & $0 \%$ & $13.3 \%$ & $86.6 \%$ & $0 \%$ & $0 \%$ \\
\hline & B & $0 \%$ & $20 \%$ & $80 \%$ & $0 \%$ & $0 \%$ \\
\hline \multirow[t]{2}{*}{ Malabaddhata } & A & $0 \%$ & $0 \%$ & $73.3 \%$ & $26.6 \%$ & $0 \%$ \\
\hline & B & $0 \%$ & 33.3 & $60 \%$ & $6.6 \%$ & $0 \%$ \\
\hline \multirow[t]{2}{*}{ Balakshaya } & A & \begin{tabular}{|l|}
$0 \%$ \\
\end{tabular} & $0 \%$ & $93.3 \%$ & $6.6 \%$ & \begin{tabular}{|l|}
$0 \%$ \\
\end{tabular} \\
\hline & B & $0 \%$ & $0 \%$ & $100 \%$ & $0 \%$ & $0 \%$ \\
\hline \multirow[t]{2}{*}{ Grahanakshaya } & $\mathrm{A}$ & $0 \%$ & $0 \%$ & $80 \%$ & $20 \%$ & $0 \%$ \\
\hline & B & $0 \%$ & $6.6 \%$ & $73.3 \%$ & $20 \%$ & $0 \%$ \\
\hline \multirow[t]{2}{*}{ Nidranasha } & $\mathrm{A}$ & $0 \%$ & $0 \%$ & $100 \%$ & $0 \%$ & $0 \%$ \\
\hline & B & $0 \%$ & $13.3 \%$ & $73.3 \%$ & \begin{tabular}{|l|}
$13.3 \%$ \\
\end{tabular} & $0 \%$ \\
\hline \multirow[t]{2}{*}{ Swasha } & A & $0 \%$ & $0 \%$ & $80 \%$ & $20 \%$ & $0 \%$ \\
\hline & B & $0 \%$ & $0 \%$ & $93.3 \%$ & $6.6 \%$ & $0 \%$ \\
\hline \multirow{2}{*}{$\begin{array}{ll}\begin{array}{l}\text { Sarvakriya } \\
\text { asamartha }\end{array} & \text { cha } \\
\end{array}$} & A & $0 \%$ & $0 \%$ & $40 \%$ & $60 \%$ & $0 \%$ \\
\hline & B & $0 \%$ & $13.3 \%$ & $80 \%$ & $6.6 \%$ & $0 \%$ \\
\hline
\end{tabular}

Table 13: Objective Parameters

\begin{tabular}{|l|l|l|l|l|l|l|}
\hline Variables & Group & CI-3 & CI-2 & CI-1 & CS & CD \\
\hline \multirow{2}{*}{ Systolic B.P } & $\mathrm{A}$ & $0 \%$ & $20 \%$ & $6.6 \%$ & $73.3 \%$ & $0 \%$ \\
\cline { 2 - 7 } & $\mathrm{B}$ & $0 \%$ & $13.3 \%$ & $13.3 \%$ & $73.3 \%$ & $0 \%$ \\
\hline \multirow{2}{*}{ Wiastolic B.P } & $\mathrm{A}$ & $0 \%$ & $0 \%$ & $26.6 \%$ & $73,3 \%$ & $0 \%$ \\
\cline { 2 - 7 } & $\mathrm{B}$ & $0 \%$ & $0 \%$ & $20 \%$ & $80 \%$ & $0 \%$ \\
\hline \multirow{2}{*}{ Hb\% } & $\mathrm{A}$ & $0 \%$ & $0 \%$ & $20 \%$ & $80 \%$ & $0 \%$ \\
\cline { 2 - 7 } & $\mathrm{B}$ & $0 \%$ & $0 \%$ & $26.6 \%$ & $73.3 \%$ & $0 \%$ \\
\cline { 2 - 7 } & $\mathrm{A}$ & $0 \%$ & $0 \%$ & $53.3 \%$ & $40 \%$ & $6.6 \%$ \\
\hline
\end{tabular}


Singh Binod Kumar et al. 'Jara' with Respect to Ageing Process with Ashwagandha Rasayana and Matra Basti

\section{Results Related for Individual Group After Treatment}

\section{Group-A}

Excellent response- No patient showed excellent response after treatment.

Good Response- In 13.3\% patients of Agmimandhya and 20\% of systolic pressure showed Good response after treatment Encouraging Response- $86.6 \%$ of Agnimandhya,73.3\% of Mala baddhata, $93.3 \%$ of Bala kshaya, $80 \%$ of Grahana kshaya, $100 \%$ of Nidra nasha $80 \%$ of Shwasa, $40 \%$ of Sarva karya aswasamarth, $6.6 \%$ of systolic blood pressure, $26.6 \%$ of diastolic blood pressure, $20 \%$ of weight and $53.3 \%$ of $\mathrm{Hb} \%$, Showed encouraging response after treatment. Clinically stable $26.6 \%$ of Mala baddhata, 46.6\% of Nidra nasha, 6.6\% of Bala kshaya, 20\% of Grahana kshaya, 20\% of Shwasa, 60\% of Sarva karya cha Aswasamarth, 73.3\% 0f systolic blood pressure,73.3\% of diastolic blood pressure, $80 \%$ of weight, $40 \%$ of $\mathrm{Hb} \%$, were clinically stable after treatment.

Deteriorated $-6.66 \%$ persons of $\mathrm{Hb} \%$ shown clinically deterioration at the end of the treatment.

\section{Group-B}

Excellent response- No patient showed excellent response after treatment.

Good Response- In 20\% patients of Agmimandhya, 33.3\% of Malabadhata, 6.6\% of Grahana kshaya, 13.3\% of Sarvakriya cha asamartha, $13.3 \%$ of systolic pressure and $6.6 \%$ of $\mathrm{Hb} \%$ showed Good response after the treatment. Encouraging Response- $80 \%$ of Agnimandhya, 60\% of Mala baddhata, $100 \%$ of Bala kshaya, 73.3\% of Grahana kshaya, 73.3\% of Nidra nasha $93.3 \%$ of Shwasa, $80 \%$ of Sarva karya cha Aswasamartha, 13.3\% of systolic blood pressure, 20\% of diastolic blood pressure, $26.6 \%$ of weight and $46.6 \%$ of $\mathrm{Hb} \%$, Showed clinically encouraging response after the treatment.

Clinically stable - 6.6\% of Mala baddhata, 20\% of Grahana kshaya, 13.3\% of Nidranasha $6.6 \%$ of Shwasa, $6.6 \%$ of Sarva karya cha Aswasamarth, 73.3\% of systolic blood pressure, $80 \%$ of diastolic blood pressure, $73.3 \%$ of weight and $46.6 \%$ of $\mathrm{Hb} \%$, were clinically stable after treatment.

Deteriorated - No patient showed clinically deterioration at the end of the treatment.

Table 14: Percentage of patients from clinical improvement of overall severity after the Treatment

\begin{tabular}{|l|l|l|l|l|l|}
\hline Group & CI-3 & CI-2 & CI-I & CS & CD \\
\hline A & $0 \%$ & $33.3 \%$ & $66.6 \%$ & $0 \%$ & $0 \%$ \\
\hline B & $0 \%$ & $100 \%$ & $0 \%$ & $0 \%$ & $0 \%$ \\
\hline
\end{tabular}

The study indicates that 33\% patients show clinically good and 66.6\% patients clinically encouraging response from Group A where $100 \%$ patients show clinically good response. The patients from any group do not show excellent response or clinically stable or clinically deteriorated response after the treatment.

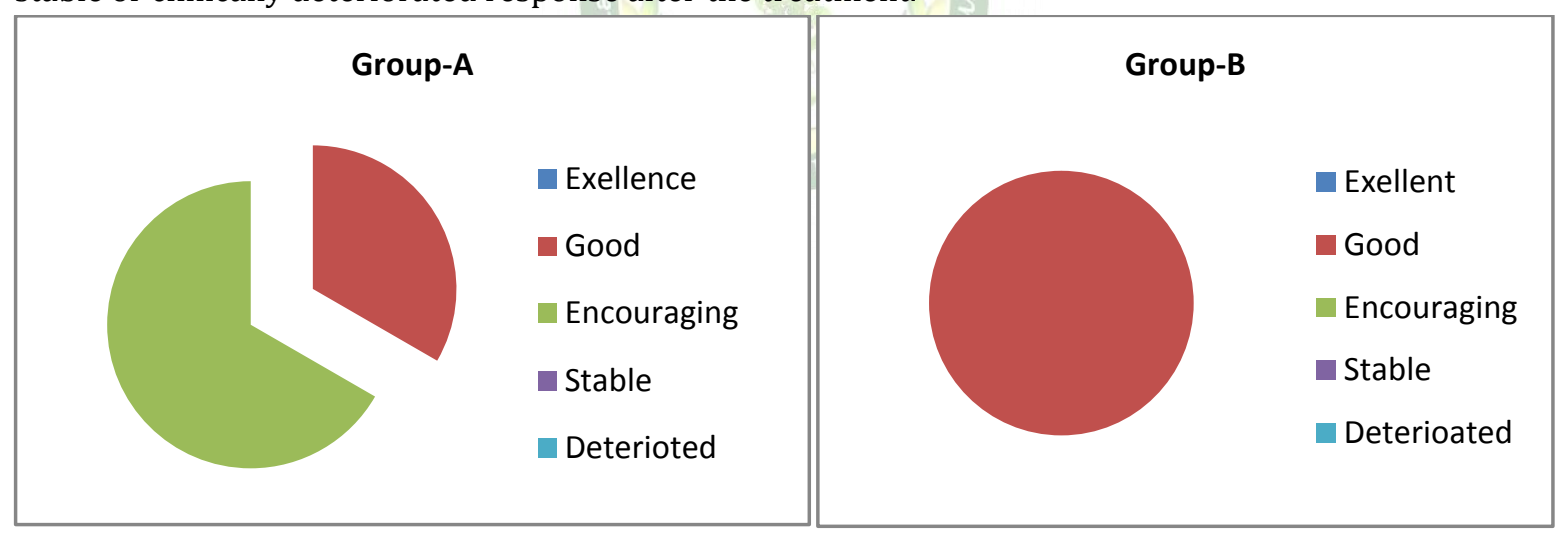

Figure - 1 showing the result of Group A

Figure- 1.1 showing the result of Group-B after Treatment after treatment

Table 15: Showing the Statistical Results of Group - A Paired Sample Test

\begin{tabular}{|c|c|c|c|c|c|c|c|c|}
\hline \multirow{3}{*}{$\begin{array}{l}\text { Variables before and } \\
\text { after treatment }\end{array}$} & \multicolumn{5}{|c|}{ Paired Differences } & \multirow[t]{3}{*}{$\mathbf{t}$} & \multirow[t]{3}{*}{ Df } & \multirow{3}{*}{$\begin{array}{l}\text { Sig.(2 } \\
\text { tailed) }\end{array}$} \\
\hline & \multirow[t]{2}{*}{ Mean } & \multirow{2}{*}{$\begin{array}{c}\text { Std. } \\
\text { Deviatio } \\
\text { n }\end{array}$} & \multirow{2}{*}{$\begin{array}{l}\text { Std. } \\
\text { Error } \\
\text { mean } \\
\end{array}$} & \multicolumn{2}{|c|}{$\begin{array}{c}95 \% \text { Confidence interval } \\
\text { of Difference }\end{array}$} & & & \\
\hline & & & & Lower & Upper & & & \\
\hline Pair 1 AgB - AgA & 1.3333 & .35187 & .09085 & .93848 & 1.32819 & 12.475 & 14 & .000 \\
\hline Pair $2 \mathrm{MaB}-\mathrm{MaA}$ & .73333 & .45774 & .11819 & .47985 & .98682 & 6.205 & 14 & .000 \\
\hline Pair 3 BaB - BaB & .93333 & .25820 & .06667 & .79035 & 1.07632 & 14.000 & 14 & .000 \\
\hline Pair 4 GrB - GrA & .80000 & .41404 & .10690 & .57071 & 1.02920 & 7.483 & 14 & .000 \\
\hline Pair 6 SwB - SwB & .80000 & .41404 & .10690 & .57071 & 1.02920 & 7.483 & 14 & .000 \\
\hline Pair 7 SKB - SKA & .40000 & .50709 & .13093 & .11918 & .68082 & 3.055 & 14 & .009 \\
\hline Pair 8 SyB - SyA & .46667 & .83381 & .21529 & .00492 & .92841 & 2.168 & 14 & .048 \\
\hline Pair 9 DyB - DyA & .26667 & .45774 & .11819 & .01318 & .52015 & 2.256 & 14 & .041 \\
\hline Pair $10 \mathrm{WtB}$ - WtA & .20000 & .41404 & .10690 & .02929 & .42929 & 1.871 & 14 & .082 \\
\hline Pair $11 \mathrm{HgB}-\mathrm{HgA}$ & .46667 & .63994 & .16523 & .11228 & .82105 & 2.824 & 14 & .014 \\
\hline
\end{tabular}


Int. J. Ayur. Pharma Research, 2016;4(8):1-11

NB: The correlation and t test of Nidranasha (Pair 5- NiB-NiA) cannot be calculated because the standard error of the difference of the parameter is 0

Table 16: Showing The Statistical Results Of Group - B Paired Sample Test

\begin{tabular}{|c|c|c|c|c|c|c|c|c|}
\hline \multirow{3}{*}{$\begin{array}{l}\text { Variables before and } \\
\text { after treatment }\end{array}$} & \multicolumn{5}{|c|}{ Paired Differences } & \multirow[t]{3}{*}{$\mathbf{t}$} & \multirow[t]{3}{*}{ Df } & \multirow{3}{*}{$\begin{array}{l}\text { Sig.(2 } \\
\text { tailed) }\end{array}$} \\
\hline & \multirow[t]{2}{*}{ Mean } & \multirow[t]{2}{*}{$\begin{array}{c}\text { Std. } \\
\text { Deviation }\end{array}$} & \multirow{2}{*}{$\begin{array}{c}\text { Std. } \\
\text { Error } \\
\text { mean }\end{array}$} & \multicolumn{2}{|c|}{$\begin{array}{c}\text { 95\% Confidence interval } \\
\text { of Difference }\end{array}$} & & & \\
\hline & & & & Lower & Upper & & & \\
\hline Pair 1 AgB - AgA & 1.20000 & .41404 & .10690 & .97071 & 1.42929 & 11.225 & 14 & .000 \\
\hline Pair $2 \mathrm{MaB}-\mathrm{MaA}$ & 1.26667 & .59362 & .15327 & .93793 & 1.59540 & 8.264 & 14 & .000 \\
\hline Pair 4 GrB - GrA & .86667 & .51640 & .13333 & .58070 & 1.15264 & 6.500 & 14 & .000 \\
\hline Pair $5 \mathrm{NiB}-\mathrm{NiA}$ & 1.00000 & .53452 & .13801 & .70399 & 1.29601 & 7.246 & 14 & .000 \\
\hline Pair 6 SwB - SwB & .93333 & .25820 & .06667 & .79035 & 1.07632 & 14.000 & 14 & .000 \\
\hline Pair 7 SKB - SKA & 1.06667 & .45774 & .11819 & .81318 & 1.32015 & 9.025 & 14 & .000 \\
\hline Pair 8 SyB - SyA & .40000 & .73679 & .19024 & .00802 & .80802 & 2.103 & 14 & .054 \\
\hline Pair 9 DyB - DyA & .20000 & .41404 & .10690 & .02929 & .42929 & 1.871 & 14 & .082 \\
\hline Pair $10 \mathrm{WtB}$ - WtA & .26667 & .45774 & .11819 & .01318 & .52015 & 2.256 & 14 & .041 \\
\hline Pair 11 HgB - HgA & .60000 & .63246 & .16330 & .24976 & .95024 & 3.674 & 14 & .003 \\
\hline
\end{tabular}

NB: The correlation and t test of Balakshaya (Pair 3- BaB-BaA) cannot be calculated because the standard error of the difference of the parameter is 0

NOTE - AgB - Agnimandha before treatment, AgA - Agnumandha after treatment, MaB - Malabaddhata before treatment, $\mathrm{MaA}$ - Malabaddhata after treatment, BaB - Balakshaya before treatment, BaA - Balakshaya after treatment, GrB - Grahana kshaya before Treatment, GrA - Grahana kshaya after treatment, NiB -Nidranasha before treatment, NiA -Nidranasha after treatment, SwB - Shwasa before treatment, SwA - Shwasa after treatment, SKB - Sarvakriya cha asamarthata before treatment, SKA - Sarvakriya cha asamarthata after treatment, SyB - Systolic blood pressure before treatment, SyA - Systolic blood pressure after treatment. DyB - Diastolic blood pressure before treatment, DyA -Diastolic blood pressure after treatment WtB - Weight before treatment, WtA - Weight after treatment, HgB Hemoglobin before treatment and HgA Hemoglobin after treatment.

Table 17: Showing the Statistical Results of Group - A after 15 days follow-up Paired Sample Test

\begin{tabular}{|c|c|c|c|c|c|c|c|c|}
\hline \multirow{3}{*}{$\begin{array}{l}\text { Variables before and } \\
\text { after treatment }\end{array}$} & \multicolumn{5}{|c|}{ Paired Differences } & \multirow[t]{3}{*}{$\mathbf{t}$} & \multirow[t]{3}{*}{ Df } & \multirow{3}{*}{$\begin{array}{l}\text { Sig.(2 } \\
\text { tailed) }\end{array}$} \\
\hline & \multirow[t]{2}{*}{ Mean } & \multirow[t]{2}{*}{$\begin{array}{c}\text { Std. } \\
\text { Deviation }\end{array}$} & \multirow{2}{*}{$\begin{array}{l}\text { Std. } \\
\text { Error } \\
\text { mean }\end{array}$} & \multicolumn{2}{|c|}{\begin{tabular}{|c|}
$95 \%$ Confidence interval \\
of Difference
\end{tabular}} & & & \\
\hline & & & & Lower & Upper & & & \\
\hline Pair 1 AgB - AgF & .60000 & .50709 & .13093 & .31918 & .88082 & 4.583 & 14 & .000 \\
\hline Pair $2 \mathrm{MaB}-\mathrm{MaF}$ & .40000 & .50709 & .13093 & .11918 & .68082 & 3.055 & 14 & .009 \\
\hline Pair 3 BaB - BaF & .13333 & .35187 & .09085 & -.06152 & .32819 & 1.468 & 14 & .164 \\
\hline Pair 4 GrB - GrF & .20000 & .41404 & .10690 & -.02929 & .42929 & 1.871 & 14 & .082 \\
\hline Pair $5 \mathrm{NiB}-\mathrm{NiF}$ & .60000 & .50709 & .13093 & .31918 & .88082 & 4.583 & 14 & .000 \\
\hline Pair 6 SwB - SwF & .13333 & .35187 & .09085 & -.06152 & .32819 & 1.468 & 14 & .164 \\
\hline Pair 7 SKB - SKF & .13333 & .35187 & .09085 & -.06152 & .32819 & 1.468 & 14 & .164 \\
\hline Pair 8 SyB - SyF & .26667 & .59362 & .15327 & -.06207 & .59540 & 1.740 & 14 & .104 \\
\hline Pair 9 DyB - DyF & .20000 & .41404 & .10690 & -.02929 & .42929 & 1.871 & 14 & .082 \\
\hline
\end{tabular}

Table 18: Showing the Statistical Results of Group - B after 15 days follow-up Paired Sample Test

\begin{tabular}{|c|c|c|c|c|c|c|c|c|}
\hline \multirow{3}{*}{$\begin{array}{c}\text { Variables before } \\
\text { and after treatment }\end{array}$} & \multicolumn{5}{|c|}{ Paired Differences } & \multirow[t]{3}{*}{$\mathbf{t}$} & \multirow[t]{3}{*}{ Df } & \multirow{3}{*}{$\begin{array}{l}\text { Sig.(2 } \\
\text { tailed) }\end{array}$} \\
\hline & \multirow[t]{2}{*}{ Mean } & \multirow[t]{2}{*}{$\begin{array}{c}\text { Std. } \\
\text { Deviation }\end{array}$} & \multirow{2}{*}{$\begin{array}{c}\text { Std. } \\
\text { Error } \\
\text { mean }\end{array}$} & \multicolumn{2}{|c|}{$\begin{array}{c}95 \% \text { Confidence } \\
\text { interval of Difference }\end{array}$} & & & \\
\hline & & & & Lower & Upper & & & \\
\hline Pair 1 AgB - AgF & .86667 & .35187 & .09085 & .67181 & 1.06152 & 9.539 & 14 & .000 \\
\hline Pair $2 \mathrm{MaB}-\mathrm{MaF}$ & 1.20000 & .56061 & .14475 & .88954 & 1.51046 & 8.290 & 14 & .000 \\
\hline Pair $3 \mathrm{BaB}-\mathrm{BaF}$ & .60000 & .50709 & .13093 & .31918 & .88082 & 4.583 & 14 & .000 \\
\hline Pair 4 GrB - GrF & .26667 & .45774 & .11819 & .01318 & .52015 & 2.256 & 14 & .041 \\
\hline Pair $5 \mathrm{NiB}-\mathrm{NiF}$ & .73333 & .45774 & .11819 & .47985 & .98682 & 6.205 & 14 & .000 \\
\hline Pair 6 SwB - SwF & .46667 & .51640 & .13333 & .18070 & .75264 & 3.500 & 14 & .004 \\
\hline Pair 7 SKB - SKF & 1.00000 & .65465 & .16903 & .63746 & 1.36254 & 5.916 & 14 & .000 \\
\hline Pair 8 SyB - SyF & .20000 & .41404 & .10690 & -.02929 & .42929 & 1.871 & 14 & .082 \\
\hline Pair 9 DyB - DyF & .13333 & .35187 & .09085 & -.06152 & .32819 & 1.468 & 14 & .164 \\
\hline
\end{tabular}


Singh Binod Kumar et al. 'Jara' with Respect to Ageing Process with Ashwagandha Rasayana and Matra Basti

Table 19: Showing the Statistical Results of Comparative Study Between Group - A and Group- B Paired Samples Test

\begin{tabular}{|c|c|c|c|c|c|c|c|c|c|}
\hline & \multicolumn{5}{|c|}{ Paired Differences } & \multirow[t]{3}{*}{$\mathbf{T}$} & \multirow[t]{3}{*}{ Df } & \multirow{3}{*}{$\begin{array}{l}\text { Sig. (2- } \\
\text { tailed) }\end{array}$} \\
\hline & & \multirow[t]{2}{*}{ Mean } & \multirow[t]{2}{*}{$\begin{array}{c}\text { Std. } \\
\text { Deviation }\end{array}$} & \multirow[t]{2}{*}{$\begin{array}{l}\text { Std. Error } \\
\text { Mean }\end{array}$} & \multicolumn{2}{|c|}{$\begin{array}{c}\text { 95\% Confidence Interval } \\
\text { of the Difference }\end{array}$} & & & \\
\hline & & & & & Lower & Upper & & & \\
\hline Pair 1 & AgA - AgB & .06667 & .25820 & .06667 & -.07632 & .20965 & 1.000 & 14 & .334 \\
\hline Pair 2 & $\mathrm{MaA}-\mathrm{MaB}$ & .26667 & .45774 & .11819 & .01318 & .52015 & 2.256 & 14 & .041 \\
\hline Pair 3 & $\mathrm{BaA}-\mathrm{BaB}$ & .06667 & .25820 & .06667 & -.07632 & .20965 & 1.000 & 14 & .334 \\
\hline Pair 4 & GrA - GrB & .06667 & .59362 & .15327 & -.26207 & .39540 & .435 & 14 & .670 \\
\hline Pair 5 & $\mathrm{NiA}-\mathrm{NiB}$ & .06667 & .25820 & .06667 & -.07632 & .20965 & 1.000 & 14 & .334 \\
\hline Pair 6 & SwA - SwB & .20000 & .56061 & .14475 & -.11046 & .51046 & 1.382 & 14 & .189 \\
\hline Pair 7 & SKA - SKB & .00000 & .75593 & .19518 & -.41862 & .41862 & .000 & 14 & 1.000 \\
\hline Pair 8 & SyA - SyB & -.06667 & .45774 & .11819 & -.32015 & .18682 & -.564 & 14 & .582 \\
\hline Pair 10 & WtA - WtB & -.13333 & 2.99682 & .77378 & -1.79292 & 1.52625 & -.172 & 14 & .866 \\
\hline Pair 11 & $\mathrm{HbA}-\mathrm{HbB}$ & .13333 & 1.06010 & .27372 & -.45373 & .72040 & .487 & 14 & .634 \\
\hline
\end{tabular}

NOTE - Ag - Agnimandhya, Ma - Malabaddhata, Ba -Balakshaya, Gr - Grahana kshaya, Ni- Nidra nasha,Sw - Shwasa, SK Sarvakriya cha asamarthata, Sy- Systolic BP, Dy -Diastolic BP, Wt -Weight, Hg- Hemoglobin, B - Before treatment, A - After treatment.

Bar Diagrams Show the Result of Group -A

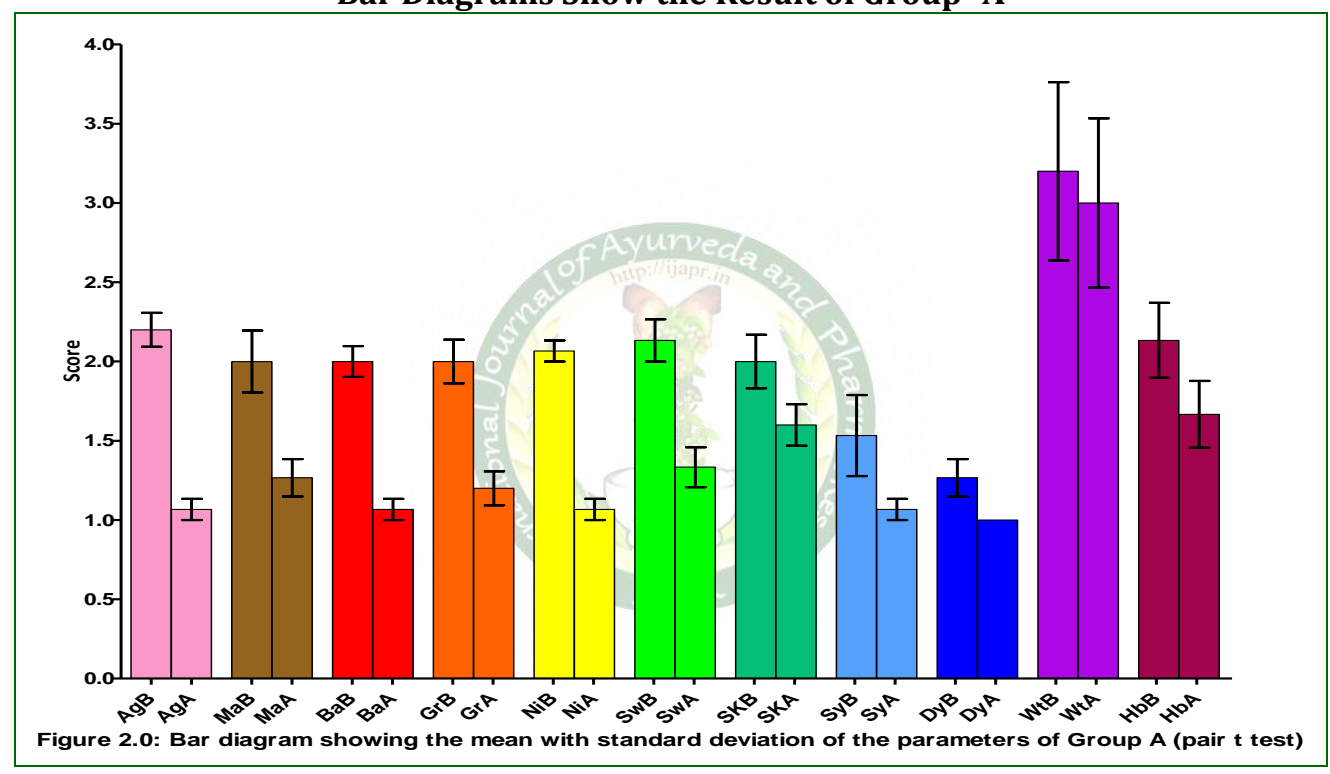

Bar Diagram Show the Result of Group - B

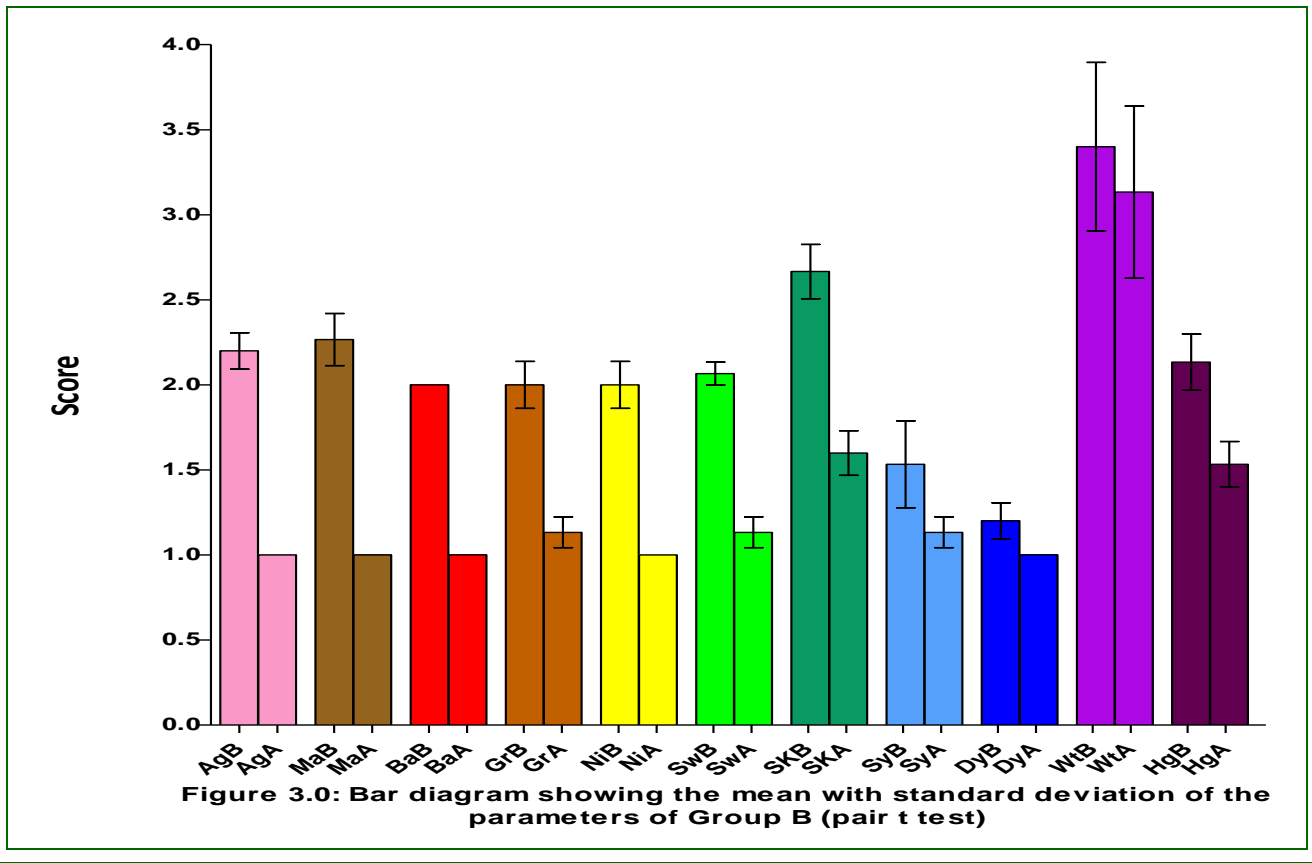


Int. J. Ayur. Pharma Research, 2016;4(8):1-11

\section{DISCUSSION ON RESULTS}

\section{Agnimandhya (Loss of appetite)}

Person of both groups A and B had shown significant result in the treatment of Agnimandhya. Group A was having t value 12.475 AT and 4.583 at FU. Group B was having $\mathrm{t}$ value $11.225 \mathrm{AT}$ and 9.539 at FU so the treatment of Group B may be better than the treatment of Group A. But after comparing the result between both Groups A and B the equal efficacy ( $p=<0.000)$ was observed on Agnimandhaya after treatment.

\section{Malabaddhata (Constipation)}

In this parameter Persons of both groups had shown significant result $(\mathrm{P}=<0.000)$. The t values of Group A were 6.205AT and 3.055 FU. The t values of Group B were $8.264 \mathrm{AT}$ and $8.290 \mathrm{FU}$ so the comparative study between Group A and Group B had shown that the treatment modality of Group B was better than Group A. $(\mathrm{p}=0.041)$.

\section{Balakshaya (Loss of physical strength)}

In the cases of Balakshaya 93.3\% case from Group A and $100 \%$ cases from Group B shown clinically encouraging response after the treatment where $6.6 \%$ of cases were normal from Group A before the treatment. The p value of both groups A and B $(<0.001)$ shown statically highly significance after the treatment so comparative study between both groups $A$ and $B$ indicates equal efficacy of both treatment modalities after the treatment.

\section{Grahana kshaya (Loss of grasping power)}

In this study, from Group A, $80.3 \%$ persons had got clinically encouraging response and $20 \%$ persons had got clinically stable response. Similarly from Group B the $6.6 \%$ persons had got clinically good response, clinically encouraging response had been seen in $73.3 \%$ persons and clinically stable seen in $6.6 \%$ persons. So both treatments modalities were capable for prevent the degeneration and strengthen the nervous system because statically the both treatment groups were highly significant. ( $p$ value $<0.001 .2$-tailed.000), but the post treatment follow up study showed t values of Group A and Group B were 1.871 and 2.256 respectively, that indicates the treatment regimen of Group B was fast acting than Group A on Grahana-kshaya.

\section{Nidra nasha (Insomnia)}

In this clinical study the both Groups A and B had shown that statically highly significant result $(p=<0.001)$ after the treatment and had equal efficacy against the Nidranasha (sleeplessness). The two weeks post treatment follow up study showed t values of Group A and Group B were 4.583 and 6.205 respectively, that indicates the treatment regimen of Group B was fast acting than Group A on Nidranasha.

\section{Shwasa (Exertional dyspnea)}

In this clinical study the both Groups A and B had shown that statically highly significant result $(p=<0.001)$ after the treatment and had equal efficacy against the Shwasa (exertional dyspnoea), but the $t$ values of two weeks post treatment follow up of Group A and Group B were 1.468 and 3.500 respectively that indicates the treatment regimen of Group B shows its efficacy in short duration of time than treatment regimen of Group A.

\section{Sarva kriya su cha asamarthata (Functional impairment)}

The $t$ values of Group A were 3.055 (AT) and 1.468 (FU) and Group B were 9.025 (AT) and 5.916 (FU) that indicates the person of Group B had shown more Significant result than the person of Group A.

\section{Discussion on Objective Variables}

\section{Blood pressure (Systolic)}

Group A had shown significant result for systolic blood pressure as Group B had shown non significant result. The t value of Group A were 2.168 (AT) and Group B was having of t value 2.103 (AT).

\section{Blood pressure Diastolic}

On this variable, Group A had shown significant result as Group $B$ had shown no significant result. The $t$ value of Group A and B were 2.256 (AT) and 1.871 (AT) respectively.

\section{Weight}

Group B had shown significant results for this parameter where Group A had shown non significant statistically. But slight increase in weight was seen in persons of group A also. The tvalue for Group A and Group B were 1.871 AT and 2.256 AT respectively.

\section{Hemoglobin}

The both Groups A and B had shown significant results. The t value for Group A was 2.824 AT and Group B was having $3.674 \mathrm{AT}$ so that indicates the treatment regimen of Group B is better than Group A but when the results were compared in between Group A and Group B statistically, it was shown equal efficacy of both Groups.

The trial drugs of group A, Ashwagandha rasayana has shown highly significant result in treating symptoms like Agnimandhya, Malabadhata, Bala kshaya, Grahan kshaya, Swasa and Nidranash similarly significant responses seen in Sarvakriyasu cha asamarthata, Blood pressure and hemoglobin percentage. Hence Ashwagandha rasayana is an effective remedy for ageing process management. The trial drug of group B, Ashwagandha rasayana with Mahanarayan tail matra basti has shown highly significant result on symptoms like Agnimandhya, Malabadhata, Bala kshaya, Grahan kshaya, Swasa, Nidranash Sarva kriya asamarthata and Hemoglobin percentage and significant responses seen on Weight loss Hence Ashwagandha rasayana with Matra basti is very effective in treating ageing ailments. In comparison in between both Group A and B, the result was statistically insignificant but the good response percentages of Group B was high than Group A.

\section{Probable action of drugs}

\section{Mode of action of Ashwagandha}

Ashwagandha by virtue of its Rasa, Guna, Veerya, Vipaka and Prabhava, promotes, digestion, metabolism, maintains homeostasis of Doshas, enriches nourishment and promotes body and mental functions thus becomes effective in aging. Its Tikta, Katu - Rasa + Laghu - Guna + Ushna veerya and Vatashamaka karma promotes digestion and metabolism their by production of rasa and 
Singh Binod Kumar et al. 'Jara' with Respect to Ageing Process with Ashwagandha Rasayana and Matra Basti

elimination of toxic metabolites and pollutants is achieved. Due to its Snigdha guna + Ushna veerya + Madhurvipaka + Vataghana + Rasayan property - Vata dosha shamana occurs. Its Snigdha guna + Madhura vipaka (Snigdha +guru) + Rasayana + Balya + Vrushya - Dhatu and Bala vardhan are achieved.

Correcting Vata + Rasayana (Prabhava) + Medhya property $>$ Does Mano Prasannata

\section{Modern concept}

Several types of alkaloids are found in the plant of which withenia, somnifera and withasomnine are important in traditional preparations. The alkaloid withaferin is responsible for the bacteriostatic and anti tumour properties. Recently steroidal factors have been isolated from leaves. The withanoloids are believed to account for the multiple applications of Ashwagandha. These molecules are steroidal and bear a resemblance, both in their action and appearance to the active constituents of Asian ginseng (panax ginseng) known as ginsenosides and are called as Indian ginseng. These serve as important hormones precursors, which the body is then able, as needed to convert into human physiological hormones. If there is an excess of certain hormones, the plant based hormones precursor occupied the so called hormone receptors sites without converting to human hormones to block absorption. In this way Ashwagandha like other adoptogenic tonic herbs is amphoteric and can serve to regulate important physiological processes, increasing or decreasing as needed. Generally Ashwagandha stimulates the immune system. It has also been shown to inhibit inflammation and improve memory. It counteracts the effects of stress and promotes wellness. Along with this, Ashwagandha improves the degenerative changes by effect on chondroplasts in cartilage as well as it creates hemopoetic effect by cyclophosphamide and reduces leukopenia ${ }^{[9]}$.

\section{Mode of action of Matra basti}

Basti Chikitsa is the prime treatment modality of Ayurveda. It is also considered as Ardha Chikitsa (half treatment). Matra basti is one of Sneha or Anuvasana Basti (unctuous enema) which promotes Bala (strength) of the person who is emaciated and debilitated. About the possibility of the absorption of Basti Dravyas (drugs) from the colon, some are of the opinion that substances other than water, salt, etc., are not absorbed from the large gut; but this is physiological phenomenon occurring in day-today life, while the colon mucosa under the effect of the medication can be rendered to absorb the unusual substance also from the large gut. Favoring this view modern medical science is suggestive of some of the nutrient enemas meant for the nutrition of the body, where absorption of carbohydrate, fat and protein is mentioned.[10] Observation of modern medical science that administration of sodium chloride improves fat absorption ${ }^{[10]}$ is curiously coinciding with the usage of salt designed by Ayurvedic medical authorities in SadyoSnehana[11] and in many Basti Dravya preparations along with the different Sneha Dravyas. Charaka narrates the role played by Lavana along with the Sneha by the words "Lavanopitaha Snehana Snehayantyachiratnaram."[12]
Charaka while assessing the Anuvasana Basti records the digestion of Sneha by the words "Sneham Pachati Pavakah"90 and after digestion Dravyas can be absorbed to cause the affect on the body.

\section{CONCLUSION}

$>$ It is interesting to note that there is a shift in the population growth from child and young to higher age groups.

$>$ Due to increased elderly population, the prevalence of geriatric specific disease conditions is also increasing. Ayurveda is essentially science of life and longevity. It presents a sound concept of aging and its prevention and management.

> The Jara vyadhi has been extensively explained in Ayurvedic classics, which can be correlated with geriatrics and Rasayana therapy acts as one of the useful remedy in promotion of the early ageing and prevention of ill effects of ageing.

$>$ Both the groups provided better results on the chief complaints But, comparison in between both the groups is insignificant, that may be due to small sample size.

\section{ACKNOWLEDGEMENT}

The study under the title of "A Comparative Clinical Study on 'Jara' with respect to Ageing Process with Ashwagandha Rasayan and Matra Basti" has been conducted by National Ayurveda Research and Training Centre Kathmandu from April 2015 and marked to be completed by November 2015. The present report is the Final Report submitted after incorporating with feedbacks and the report submitted on 29 Nov 2015. We hope that this work is highly useful for Government's policy maker, researchers, doctors and scholars of Ayurveda to framing the line of treatment for Geriatric problems. So on this occasion we express our sincere gratitude and thanks to Prof. Dr. Ramesh Kant Adhikari, Former Dean of IOM, Chairman of Ethical Committee, National Ayurveda Research and Training Centre, for continuous inspiration and valuable support to the research in Ayurveda. We would like to thanks to Dr. Shyam Babu Yadav, Executive Director, National Ayurveda Research and Training Centre for providing facilities for this research work. We are highly thankful to Prof. C. K. Gurung, Dr. T. R. Panthi and Dr. Sarita Shrestha for their valuable suggestions. We thank to all doctors, technical and non technical staffs, residents and nursing staffs of T.U Ayurveda Teaching Hospital, Kirtipur for their co-operation throughout the work. Lastly we are thankful to all patients of trial drug and all those person who have helped us directly or indirectly for this work.

\section{REFERANCES}

1. Yadav Raj Kumar, Ageing population in Nepal, Challenges and Management, Academic Voices, A Multidisciplinary Journal, Volume 2, N0. 1, 2012.

2. Senior Citizen Act, 2063 BS (2006 AD), Government of Nepal.

3. Developing Information Base On Elderly Population of Nepal - A report submitted to WHO, by Nepal Public Health Foundation (NPHF) July 2013. 
4. Adhikari S. Health, Nutrition and Care for Senior Citizens of..... JHAS, 2013, Vol. 3, No. 1 P 73-75.

5. César GG, Sergio SG, Teresa JC, Oscar RC, Luis M GR, Carmen GP. Health care utilization in the elderly Mexican population: Expenditures and determinants. BMC Public Health. 2011; 11:192.

6. Dr. Brahmanand Tripathi, Charaka Samhita vol 2 chikitsa sthan 1/1 7-8 of Agniveshkrut Charaka Drudhabala Partisamskruta, Chaukhambha Subharatai Prakashana Varanasi 2008. P 5.

7. Sri Bhava mishra, Bhava prakasha Nighantu, Guduchyadi varga 189-191, commented by Chunekar K.C. Chowkhamba Bharati Academy Varanasi 2006. P 393.

8. Ayurvedic management of selected Geriatric disease conditions, Published by Central council for Research in Ayurveda and Siddha, New Delhi p. 41.
9. Clinical evaluation of Ashokarishta, Ashwagandha Churna and Praval Pishti in the management of menopausal syndrome - Manasi B. Modi et all Ayu 2012 Oct;33(4):511-6. doi: 10.4103/0974-8520. 110529.

10. Chattergee CC.. Human Physiology; 10th ed. Vol. 1. Calcutta: Medical Allied Agency; 1985 pp. 6-36. (235).

11. Vagbhata. Ashtanga Hridaya, Sutra Sthana, Snehavidhi Adhyaya, 16/43. In: Anna Moreswara Kunte., Late. Dr, editor. Varanasi: Chaukhambha Publication; 1998. p. 536.

12. Agnivesha, Charaka, Dridhabala. Charaka Samhita, Sutra Sthana, Snehadhyaya 13/98. In: Tripathi B, Deshpande PJ, editors. reprint ed. Varanasi: Chaukambha Sanskrit Pratishtan; 2003. p. 284.

\section{Cite this article as:}

Singh Binod Kumar, Bharkher D.L. A Comparative Clinical Study on 'Jara' with Respect to Ageing Process with Ashwagandha Rasayana and Matra Basti. International Journal of Ayurveda and Pharma Research. 2016;4(8):1-11.

Source of support: Nil, Conflict of interest: None Declared
*Address for correspondence Dr Binod Kumar Singh

Asst. Professor \& Head,

Dept. of Panchakarma, Ayurveda Campus, IOM, Tribhuvan University, Kathmndu, Nepal,

Email: drbinodbaghel@yahoo.com $\mathrm{Ph}:+977-9851094371$ 